\title{
TARMED-Info
}

\section{Bericht Nr. 9}

\section{H. H. Brunner}

La versione italiana sarà

pubblicata nel no 1-2/2003

\section{UV/IV/MV-Arztvertrag/-tarif (blauer Tarif)}

Dem mit Datum vom 28. Dezember 2001 vereinbarten neuen blauen Tarif bzw. dem Rahmenvertrag wurde in der Urabstimmung vom 4. März 2002 zugestimmt. Die Vorarbeiten sind soweit abgeschlossen, dass der Tarif auf 1. Mai 2003 basierend auf der Version TARMED 1.1.r in Kraft gesetzt wird.

\section{Anmerkungen}

- Für das Jahr 2003 gilt der neue blaue Tarif ausschliesslich im ambulanten Bereich, nicht in den Spitälern (ambulant oder stationär). Dies entspricht der Beurteilung, dass der Tarif wegen für den Spitalbereich ungelöster Probleme nicht einführungsreif ist.

- Seitens der Ärzteschaft wurde immer wieder gewünscht, dass die TARMED-Tarife im Sinne eines Pilotversuches in einem Segment eingeführt werden sollten; diesem Anliegen wird mit Einführung des blauen Tarifs im UV/MV/IV-Bereich entsprochen, dem für die Einkommen der meisten Fachdisziplinen nicht vitale Bedeutung beikommt.

- Nach wiederholt bestätigten Analysen bergen die TARMED-Tarife für die Radiologen die Gefahr exzessiver, eindeutig > 10\% liegender Einkommensverluste. Diese werden durch in einer speziellen Zusatzvereinbarung geregelte Massnahmen zeitverzugslos korrigiert werden können. Für Fachdisziplinen, die ähnliche Gefahren reklamieren, aber nicht, wie die Radiologen, belegen können, wurde eine spezielle Observation vereinbart, die bei ähnlichen akut einsetzenden Einkommensveränderungen schnell Platz greifen würde (Allergologie und Immunologie, Kinder- und Jugendpsychiatrie und -psychotherapie, Kinderchirurgie, Neuropädiatrie, Orthopädische Chirurgie). Generell wurde festgelegt, dass alle Fachdisziplinen in der Einführungsphase 2003/04 einem speziellen Monitoring unterworfen werden. Wir halten fest, dass mit Einführung des blauen Tarifs solche Einkommenseinbussen durch Zahlen belegt werden müssen; institutionell sind heute die Voraussetzungen für solche Analysen gegeben.
- Seitens verschiedener Softwarehäuser wie auch ihrer Kunden wurde immer wieder geklagt, die «Informatikvoraussetzungen» zur Einführung seien nicht gegeben. Hierzu halten wir folgende Punkte fest:

- Die Tarifstruktur TARMED ist seit dem Jahre 2000 allen Softwarehäusern bekannt; sie wurde im Kern nicht mehr verändert. Version 1.1 wie auch Version 1.1.r sind nur Updates dieser Tarifstruktur.

- Bekannterweise wurde die Einführung von TARMED seitens einiger Softwarehäuser mit genereller Softwareanpassung und anderen ausserhalb TARMED liegenden Problemen verknüpft. Dies ist nicht dem TARMED-Projekt anzulasten und kann nicht als Argument für einen weiteren Aufschub der Einführung ins Feld geführt werden.

- Ebenso bekannterweise sind Softwarehäuser vorbereitet, TARMED einzuführen; ihre Dienste können im Sinne des auch hier geltenden freien Wettbewerbs in Anspruch genommen werden.

- Immer wieder geistern Gerüchte durch die Ärzteschaft, zur Abrechnung gemäss TARMED seien sogenannte Validatoren vonnöten. Dies trifft nicht zu. Validatoren können in Zukunft eine Rolle spielen; seitens der FMH werden aber nur Validatoren anerkannt, die durch die FMH akzeptiert wurden. Sollten seitens der Versicherer andere Validatoren eingesetzt werden, so würden die Ärzte unverzüglich nur noch in Printform abrechnen. Ergänzend sei erwähnt, dass vertraglich nur der reine XML-Standard ohne jegliche Zusätze bzw. Weiterentwicklungen vereinbart wurde; Anwendungen anderer Standards wären als Vertragsbruch zu qualifizieren.

- Die reale Anwendung der TARMED-Tarifstruktur wird eine Unzahl von Fehlern und Inkonsistenzen offenlegen. Diese würden allenfalls provisorisch korrigiert, auf jeden Fall aber zur Korrektur der Tarifstruktur in die entsprechenden Verhandlungen eingebracht werden. 


\section{Einführung TARMED im KVG-Bereich bzw. in den Spitälern}

Die TARMED-Tarife im KVG-Bereich (für Arztpraxen pro Kanton) und im Spitalbereich sollen bis spätestens 1. Januar 2004 erfolgen. Die Koordination auf schweizerischer Ebene erfolgt durch die sogenannte Gruppe G7, zusammengesetzt aus Vertretern der kantonalen Ärztegesellschaften und der FMH.

\section{Anmerkungen}

- Die kantonalen Einführungsverträge inkl. kantonalem Start-TPW (Starttaxpunktwert) für die Arztpraxis sollen bis zum 2. Quartal 2003 ausgehandelt sein. Die Spitäler müssen im selben Zeitraum ihre Verträge aushandeln, soll das ambitiöse Ziel 1. Januar 2004 erreicht werden.

- Nur in Konturen erkennbar ist, wie im Rahmen des sogenannten Projektes LOA Margen aus SD in TPW erfolgen soll. Ebenso unklar ist, gerade hier aber von zentraler Bedeutung, die Frage wie und wann die Interventionen der Preisüberwachung erfolgen werden. Offen bleibt ebenso - obwohl immer wieder von seiten der FMH moniert - die Frage, wie Rekurse gegen die Starttaxpunktwerte die Einführung behindern werden, insbesondere im Lichte der Frage, ob solchen Klagen rechtsaufschiebende Wirkung beikommen wird. Die Aktivitäten der zuständigen staatlichen Instanzen, in diesem zentralen Punkt Klarheit zu schaffen, haben bis jetzt nicht gerade einen fanatischem Tatendrang an den Tag gelegt.

- Die leitenden Ärztinnen und Ärzte an öffentlichen Spitälern werden sich durch die Einführung der TARMED-Tarife, in Kombination mit absehbaren Änderungen des KVG (Wegfall der Privatabteilung, Wegfall von sogenannten Privattarifen) sowie Rückgang der Anzahl Halbprivat- und Privatversicherungen, mit besonderen Problemen konfrontiert sehen. Die Spitaldirektionen bzw. die hinter ihnen stehenden Gesundheitsdirektionen scheinen in Mehrheit forciert ihre institutionellen Interessen zu vertreten; die leitenden Spitalärztinnen und -ärzte, mit Tariffragen weit weniger vertraut als ihre Praxiskollegen, stehen diesen Entwicklungen recht hilflos gegenüber. Die FMH kann diese Ärztegruppe nur einmal mehr ihrer Unterstützung versichern ..., die aber von den Betroffenen auch gewünscht werden muss.

\section{Abschluss des Projektes TARMED / Übergang der Arbeiten an die NFO}

Das Projekt TARMED wird per 31. Dezember 2002 beendet; noch offene Probleme werden der Nachfolgeorganisation (NFO), der TARMED Suisse, übergeben, die mit der Wartung und Weiterentwicklung der Tarifstruktur betraut wird. TARMED Suisse wird von FMH, H+, MTK (eidgenössische Sozialversicherer) und santésuisse getragen, mit der SDK im Beobachterstatus.

\section{Anmerkungen}

- Die Reengineeringarbeiten und -verhandlungen sind aus Sicht der FMH nur teilweise erfolgreich verlaufen (eine detaillierte Aufstellung befindet sich unter www.fmh.ch). Die Tarifstruktur konnte nach wie vor nicht für die Einführung im Spital aufbereitet werden. Die FMH gibt ihrer bestimmten Hoffnung Ausdruck, dass diese Tarifstruktur bis zur Einführung im Spitalbereich noch «fit» gemacht werden kann und die Korrekturen von RE II in die Einführungsstruktur eingebaut werden. Dies ist umso mehr möglich und gerechtfertigt, weil die Einführung im KVG-Bereich bzw. in den Spitälern mindestens bis 1. Januar 2004 verzögert wird.

- Seit der Zeit des GRAT-Projektes ist bekannt, dass eine adäquate Abgeltung der Belegärzte im Rahmen der Tarifstruktur GRAT/TARMED nur mit grossen Mühen erreichbar sein würde. Obwohl gewisse Verbesserungen in der TARMEDTarifstruktur erreicht werden konnten, vermögen auch diese die Probleme nicht grundsätzlich zu lösen. Dies wird nur mittels eines auf TARMED aufsetzenden Tarifs für Belegärzte möglich sein, dessen Aufbau durch eine Arbeitsgruppe FMHSVBP-FMS an die Hand genommen wird.

- Der Tarifdienst der FMH wurde als Unterstützungsstruktur für die Verhandlungen GRAT-TARMED aufgebaut. Mit Abschluss des TARMED-Projektes ist nun auch der Zeitpunkt gekommen, an dem der Tarifdienst grundsätzlich neu strukturiert und ausgerichtet werden wird. Die entsprechenden Beschlüsse, vor allem auch hinsichtlich Finanzierung, werden durch die ordentliche Ärztekammer 2003 gefasst werden müssen. Im Rahmen dieser Neuausrichtung sollen auch die kantonalen Aktivitäten mit denjenigen der FMH «unter einem gemeinsamen Dach» integriert werden. Die Entwürfe können unter www.fmh.ch eingesehen werden. 


\section{Dignitätserhebung}

Die TARMED-Tarifstruktur baut auf einer Zuordnung der Leistungen an Gruppen von Ärztinnen und Ärzten auf, die kraft ihrer Aus- und Weiterbildung (Facharzttitel, Fähigkeitsausweis) zur Abrechnung berechtigt sind (sogenannte qualitative Dignität). Diese Berechtigungen werden durch eine sogenannte Dignitätserhebung definiert, die bei der grossen Mehrzahl der Schweizer Ärztinnen und Ärzte durchgeführt werden wird.

\section{Anmerkungen}

- Die Dignitätserhebung wird ab 15. März 2002 bei allen FMH-Mitgliedern durchgeführt. Über diese Dignitätserhebung wird detailliert informiert werden.

- Im Rahmen der Dignitätserhebung erhält jeder Arzt/jede Ärztin eine EAN-Nummer zugeteilt, die unter anderem die Berechtigung zur Abrechnung von Leistungen zulasten der Sozialversicherer ausweisen wird.
- In steter Wiederholung betonen wir, dass die sogenannte quantitative Dignität für den einzelnen abrechnenden Arzt bedeutungslos ist. Die sogenannte Besitzstandsgarantie, d. h. Leistungen ausserhalb des durch Facharzttitel bzw. Fähigkeitsausweise definierten Bereichs als Übergangslösung von 3 Jahren abrechnen $\mathrm{zu}$ dürfen, wurde vertraglich verankert. Sie kann weiterdauern, wenn der Arzt/die Ärztin eine regelkonforme Fortbildung in diesen Gebieten absolviert. Wir bitten die Fachgesellschaften, Fortbildungsveranstaltungen auch für Ärztinnen und Ärzte anzubieten bzw. diese zu öffnen -, die nicht Facharzttitelträger oder Besitzer eines Fähigkeitsausweises sind; sollte dies nicht geschehen, so müsste die FMH im Sinne einer Gewährleistung der Ärztekammerbeschlüsse solche Fortbildungsveranstaltungen ohne Mitwirkung der entsprechenden Fachgesellschaften organisieren, eine Entwicklung, die niemand im Ernst wollen kann. 